\title{
AVALIAÇÃO DO ENRAIZAMENTO DE ESTACAS DE CRISÂNTEMO (Chrysanthemum morifolium L.) CV. WHITE REAGAN 606 TRATADAS COM ÁCIDO INDÓLBUTÍRICO (IBA)
}

\author{
F.L.CUQUEL ${ }^{1}$; N. do P. GRANJA $;$ K. MINAMI ${ }^{3}$
}

\begin{abstract}
RESUMO: Avaliou-se o efeito de doses crescentes do ácido indolbutírico (IBA) e de diferentes tempos de imersão no enraizamento de estacas de Chrysanthemum morifolium cv. White Reagan 606 . $O$ fator doses foi aplicado em quatro níveis: $0,500,1000$ e $1500 \mathrm{ppm}$ de IBA e o fator tempo em três níveis: $5 \mathrm{~s}, 1 \mathrm{~h}$ e $2 \mathrm{~h}$. $O$ delineamento utilizado foi o de blocos completos casualizados com parcelas subdivididas, onde as dosagens de IBA foram aplicadas como tratamento principal. $O$ experimento foi instalado em caixas de isopor, em casa de vegetação sob nebulização, tendo vermiculita como substrato. $O$ momento adequado para o transplante foi avaliado atraves da leitura de uma repetição adicional utilizada como controle. Obteve-se enraizamento em todas as combinaçōes de tratamento, mesma na ausência de IBA. De maneira geral o tratamento rápido tende a ser mais facilmente eficiente quando combinado com as maiores dosagens, enquanto os tratamentos mais demorados com as menores dosagens.
\end{abstract}

Descritores: crisântemo, Chrysanthemum morifolium, enraizamento de estacas, ácido indolbutírico (IBA).

\section{ROOTING EVALUATION IN Chrysanthemum morifolium CV. WHITE REAGAN 606 STEM CUTTINGS AS AFFECTED BY INDOLBUTYRIC ACID (IBA)}

\begin{abstract}
The effect of rising concentrations of indolbutyric acid (IBA) solutions was evaluated with different exposition times in stem cuttings of Chrysanthemum morifolium cr. White Reagan 606. The applied concentrations were $0,500,1000$ and $1500 \mathrm{ppm}$ of IBA during $5 \mathrm{~s}, 1 \mathrm{~h}$ and $2 \mathrm{~h}$, in a randomized complete split-plot block design, with IBA concentrations as main treatment. The experiment was installed in styrofoam trays in a green-house with a mist device and vermiculite as substrate. Transplanting time was determined by an additional block. All treatments induced rooting even in the absence of IBA. In a general sense the fastest treatments tended to be the more efficient when combined with higher concentrations.
\end{abstract}

Key Words: Chrysanthemum morifolium, stem rooting, indolbutyric acid (IBA).

\section{INTRODUÇÃO}

O crisântemo, Chrysanthemum morifolium, tem sua origem no Extremo Oriente, sendo cultivado como planta ornamental há mais de 1.200 anos na China e no Japão. Destaca-se atualmente em nível mundial entre as espécies ornamentais de importância econômica.

O seu cultivo encontra-se estabelecido no Brasil há cerca de 50 anos, tendo particular expressão nos Estados de São Paulo, Rio de Janeiro e Minas Gerais. Constitui-se numa das principais fontes de renda para parte da população rural da Grande São Paulo e de outros municípios relativamente próximos à capital como Atibaia, Campinas, Ibiúna, Jaguariúna, Jacareí, Itaquaquecetuba, São Roque e Tatuí.

O retorno financeiro obtido com a exploração da produção e comércio de plantas ornamentais, muito tem contribuído para o estabelecimento de técnicas adaptadas à condições particulares de cultivo.

São Paulo é o principal centro brasileiro para a comercialização de ornamentais. Durante os últimos dez anos, os crisântemos tem ocupado a principal posição no que diz respeito ao volume de flores

\footnotetext{
${ }^{1}$ Engenheiro Agrônomo, Secretaria da Agricultura/ITCF/PR. Bolsista do CNPq.

${ }^{2}$ Engenheiro Agrônomo, Seção de Raízes e Tubérculos. Instituto Agronômico/Campinas,SP. Bolsista da CAPES.

${ }^{3}$ Engenheiro Agrônomo, Professor Titular do Departamento de Horticultura/ESALQ/USP, Piracicaba-SP.
} 
comercializadas no CEAGESP (CEAGESP, 1989).

Mais recentemente, o mercado de exportação tem se mostrado amplamente favorável aos produtos brasileiros, tanto a produção de flores quanto aos materiais de propagação, em função do inverno europeu, o que exige desses produtores um grau de conhecimento tecnológico ainda maior, especialmente no que diz respeito aos aspectos fitossanitarios e de padronização.

Apesar da existencia desse quadro extremamente favorável, a pesquisa ainda não dispõe de algumas informações importantes para a otimização da produção de crisântemos, principalmente se levarmos em consideração as exigências ao nível de cultivar. Um desses pontos, diz respeito ao processo de enraizamento de estacas, fundamental por sua vez para o estabelecimento de melhores culturas, mais precoces e uniformes.

Este experimento visa avaliar o enraizamento deestacas de crisântemos do cultivar White Reagan 606 tratadas com ácido indolbutírico, técnica usualmente utilizada pelos produtores de plantas ornamentais em diferentes veiculações, doses e formas de tratamento.

\section{REVISÃO BIBLIOGRÁFICA}

A propagação de crisântemos pode ser realizada através das sementes, por rebentos ou por estacas.

A propagação por sementes exige tratos culturais e fitossanitários muito trabalhosos e de alto risco. Além disso, a expressão da variabilidade genética existente mostra-se incompatível com o grau de padronização exigido pelo mercado. É utilizada apenas no processo de melhoramento.

A multiplicação por rebentos é mais demorada e pela tendência ao acúmulo de patógenos no material de propagação, formam-se plantas com tendência à "degeneração", isto é, redução da produtividade devido ao acúmulo de patógenos no material de propagação. Segundo LEMAIRE (1964) a multiplicação por estacas é a mais fácil, desde que se possuam boas matrizes.

As estacas são separadas da planta-matriz através de um corte liso, preferencialmente próximo e abaixo de uma gema, com 5 a $7 \mathrm{~cm}$ de comprimento (LEMAIRE, 1964). Alguns produtores de estacas ligados à cooperativa Holambra I, Jaguariúna-SP, as agrupam em lotes com peso homogêneo entre 0,7 e 1,2 $g$ por estaca, visando a obtenção de stands mais homogêneos.

Segundo ADRIANCE \& BRISON (1939) estacas herbáceas, como as do crisântemo, são principalmente cultivadas em estufas. Deve-se evitar extremos de temperatura, enquanto a manutenção da umidade relativa do ar em níveis elevados evita a desidratação do material de propagação (HARTMANN \& KESTER, 1976), favorecendo ainda a hidratação do substrato, essencial para induzir a formação de raízes (CUTTER, 1986). A luz deve incidir de forma difusa sobre as estacas, uma vez que a luz direta inibe a formação de raízes (JANICK, 1968).

TAKEYOSHI et al. (1983) utilizando diferentes substratos (casca de arroz, solo + areia + esterco, vermiculita + solo, vermiculita fina, vermiculita grossa e vermiculita + palha de arroz), não observaram diferenças significativas quanto ao enraizamento de estacas de crisântemo. O enraizamento necessário para o transplante foi obtido entre 12 e 14 dias após o estaqueamento, quando as raízes alcançaram entre 1,5 a 2,0 cm de comprimento.

Nos sistemas de produção especializados, a fase de enraizamento é agilizada ao máximo, visando a redução dos custos diretos da produção, que pelas condições controladas normalmente empregadas, são bastante altos nessa fase. Nessas condições, consegue-se enraizamento satisfatório em até 8 dias.

Antes do plantio, as estacas são tratadas com reguladores de crescimento para aumentar a porcentagem, velocidade, qualidade e uniformidade de enraizamento (HARTMANN \& KESTER, 1976).

Os produtores paulistas utilizam-se frequentemente do ácido indolbutírico (IBA) veiculado em talco, à concentração de 1.000 a 1.500 ppm, tratando apenas a base da estaca, plantando em seguida ou armazenando em câmara fria e úmida, $2 \mathrm{a}^{\circ} \mathrm{C} \mathrm{e}$ $85 \%$ UR, por até 20 dias.

WODECKI \& HOLCOMB (1989) obtiveram aumento da velocidade para o enraizamento de estacas de crisântemos com o tratamento rápido $(5 \mathrm{~s}) \mathrm{em}$ solução de $1.000,2.000$ e $4.000 \mathrm{ppm}$ de IBA. Dentre esses, o tratamento a $1.000 \mathrm{ppm}$ levou à maior precocidade.

SAMANANDA et al. (1972), estudando o uso conjunto do IBA e do etileno (ácido 2-cloroetil fosfórico), observaram que enquanto o IBA favoreceu a indução de raízes em estacas de crisântemo, 0 etileno teve ação favorável ao crescimento dessas raízes. Maior número de raízes foram formadas pelo tratamento rápido em solução a $1.000 \mathrm{ppm}$ de IBA. Os mesmos autores classificam os cultivares estudados quanto à maior ou menor facilidade para o enraizamento, reforçando a necessidade de estudos específicos. 


\section{MATERIAL E MÉTODOS}

O experimento foi conduzido na Estação Experimental da ESALQ - Piracicaba, durante o mes de setembro de 1990.

Nesse período a temperatura máxima é de $27,6^{\circ} \mathrm{C}$ em média, enquanto as mínimas se situam ao redor de $13,2^{\circ} \mathrm{C}$. A precipitação média é de $61 \mathrm{~mm}$ e a umidade relativa do ar $63 \%$ conforme dados da Estação Climatológica da ESALQ/USP acumulados nos últimos 20 anos.

Para o estaqueamento, utilizou-se bandejas de isopor com 72 células com $12 \mathrm{~cm}$ de altura, próprias para o enraizamento, tendo vermiculita de textura fina como substrato. Conduziu-se o experimento em casa de vegetação com telhado translúcido e ripado nas laterais, equipado com sistema nebulizador automático.

Utilizou-se o cultivar White Reagan 606, produzido e exportado por KLASS SCHOENMAKER \& FILHOS (Fazenda Universo, Santo Antonio da Posse-SP).

Estudaram-se quatro tratamentos em solução aquosa, sendo três deles com 500,1.000 e 1.500 ppm de IBA, além da testemunha sem IBA. Testaram-se também três tempos de exposição ao produto: tratamento rápido (cerca de $5 \mathrm{~s}$ ), uma e duas horas de imersão da base das estacas.

O delineamento experimental utilizado foi o de blocos completos casualizados com parcelas subdivididas. As dosagens de IBA foram aplicadas às parcelas como tratamento principal e os tempos de imersão aplicados às sub-parcelas como tratamento secundário, num total de 4 blocos.

As dosagens de IBA em 4 níveis $(0,500$, 1.000 e $1.500 \mathrm{ppm}$ ) e os tempos de imersão em 3 niveis (5s, 1h, 2h), totalizaram 12 combinações que consumiram 144 estacas em cada um dos 4 blocos. Foram ainda plantadas 240 estacas que receberam os mesmos tratamentos do experimento, destinadas a avaliar a evolução do enraizamento, através de protocolos semanais em grupos de 4 estacas.

Encerrou-se o experimento quando um desses grupos apresentou aspecto visual adequado, com raízes em média superiores a $2 \mathrm{~cm}$.

Cada sub-parcela, composta por 12 estacas, teve 6 plantas avaliadas quanto ao seu aspecto visual, recebendo uma nota entre 0 (péssimo) e 5 (excelente), individualmente, por 4 observadores. Mediu-se também o comprimento da maior raíz e determinou-se o peso seco das mudas enraizadas em estufa a $60^{\circ} \mathrm{C}$.

\section{RESULTADOS E DISCUSSÃO}

$\mathrm{Na}$ colheita realizada 18 dias após o estaqueamento, avaliou-se o peso seco das mudas, o comprimento da maior raiz e a soma de 4 notas entre 0 e 5 para $o$ aspecto visual das mudas. A variável nota foi transformada em raiz quadrada de $X+0,5$ para atender às exigências da análise da variância (ANAVA).

A ANAVA indicou para as 3 variáveis a existência de diferenças significativas para doses de IBA e tempos de tratamento, assim como suas interações, de acordo com a Tabela 1 . Tais resultados são de certa forma esperados em experimentos exploratórios como este, justificados aqui pelo reduzido volume de informações disponível, especialmente nas condições brasileiras.

Optou-se pelo estudo dos efeitos das interações entre tratamentos, em virtude das suas significâncias, o que torna sem sentido o estudo isolado dos efeitos das doses de IBA e dos tempos de imersão.

$O$ desdobramento das interações determinou para a maioria dos tratamentos a existência de efeitos significativos tanto entre doses de IBA dentro de tempos de imersão (Tabela 2), quando para tempos de imersao dentro de doses (Tabela 3), observando-se coeficientes de determinação sempre elevados em todos os casos.

Os efeitos de tempo de imersão dentro de doses de IBA (Figura 1) assim como os efeitos de doses de IBA dentro de tempos de imersão (Figura 2), mostram comportamento similar entre as variaveis notas e comprimento da maior raíz, conduzindo sempre as mesma conclusões. Observa-se ainda menores coeficientes de variação (Tabela 1) para a variavel notas, fato que aliado a sua maior praticidade, motivo, a discussão em torno da variavel notas.

Para a variavel notas, Tabela 2, Figura 1, observou-se um efeito crescente do tempo de imersão em água, na ausência de IBA, com máximo em 1,3 h de imersão. Na presença de IBA, o aumento do tempo de imersão ocasionou efeitos linearmente decrescentes para as três dosagens estudadas.

Para a variavel comprimento da maior raiz, os resultados seguiram tendências semelhantes ãs observadas para a variavel notas, com efeito do tempo de imersão em agua, sem IBA, linearmente crescente.

O peso seco das mudas mostrou efeitos genericamente decrescentes para tempo de imersão na presença de IBA, principalmente nas duas maiores dosagens testadas, à semelhança do que foi observado para notas e comprimento da maior raiz. 
Tabela 1- Resumo dos principais resultados da análise da variância, respectivas médias gerais e coeficientes de variação.

\begin{tabular}{|c|c|c|c|c|}
\hline \multirow[t]{2}{*}{ Variável } & \multirow[t]{2}{*}{ Fonte de Variação } & \multicolumn{3}{|c|}{ Média } \\
\hline & & $\mathbf{F}$ & $\mathrm{CV} \%$ & Geral \\
\hline \multirow[t]{3}{*}{ Peso Seco } & Doses de IBA & $10,30 * *$ & $9,62(a)$ & $1,76(\mathrm{c})$ \\
\hline & Tempo de Tratamento & $8,20 * *$ & $10,33(b)$ & \\
\hline & Interação & $3,39 * *$ & & \\
\hline \multirow{3}{*}{$\begin{array}{l}\text { Comprimento } \\
\text { da maior raíz }\end{array}$} & Doses de IBA & $24,98 * *$ & $21,06(a)$ & $19,60(d)$ \\
\hline & Tempo de Tratamento & $8,89 * *$ & $24,65(\mathrm{~b})$ & \\
\hline & Interação & $4,88 * *$ & & \\
\hline \multirow[t]{3}{*}{ Soma de Notas } & 4 Doses de IBA & $56,76 * *$ & $12,82(a)$ & $3,04(c)$ \\
\hline & Tempo de Tratamento & $38,72 * *$ & $13,16(b)$ & \\
\hline & Interação & $13,40 * *$ & & \\
\hline
\end{tabular}

** Significativo ao nível de $99 \%$ de probabilidade

(a) Ao nível de parcelas; (b) Ao nível de sub-parcelas; (c) Gramas por 6 mudas; (d) Milímetros;

(e) Soma de notas transformada em raíz quadrada de $x+0,5$

Tabela 2 - Efeito de tempos dentro de doses de IBA para as variáveis estudadas.

\begin{tabular}{||cccl||}
\hline Desdobramento & $\mathrm{F}$ & $\mathrm{r}^{2}$ & Equação Ajustada \\
Var. Peso Seco & & & \\
Dose 0 (Tempos) & $2,32^{(1)}$ & 1,000 & $\mathrm{Y} 1=1,590+0,255(\mathrm{X})-0,110(\mathrm{X} 2)$ \\
Dose 500 (Tempos) & $21,71^{* *}$ & 1,000 & $\mathrm{Y} 2=1,790+0,746(\mathrm{X})-0,336(\mathrm{X} 2)$ \\
Dose 1000 (Tempos) & $7,02^{*}$ & 1,000 & $\mathrm{Y} 3=1,873+0,226(\mathrm{X})-0,191(\mathrm{X} 2)$ \\
Dose 1500 (Tempos) & $6,72^{* *}$ & 0,950 & $\mathrm{Y} 4=1,881-0,252(\mathrm{X})$ \\
Var. Comp. Raízes & & & \\
Dose 0 (Tempos) & $4,80^{(2)}$ & 0,999 & $\mathrm{Y} 1=22,58+3,750(\mathrm{X})$ \\
Dose 500 (Tempos) & $3,61^{(3)}$ & 0,862 & $\mathrm{Y} 2=25,75-3,250(\mathrm{X})$ \\
Dose 1000 (Tempos) & $19,85 * *$ & 0,996 & $\mathrm{Y} 3=24,21-7,625(\mathrm{X})$ \\
Dose 1500 (Tempos) & $17,94 * *$ & 1,000 & $\mathrm{Y} 4=20,25-7,250(\mathrm{X})$ \\
Var. Notas & & & \\
Dose 0 (Tempos) & $6,09 *$ & 0,583 & $\mathrm{Y} 1=3,721+0,322(\mathrm{X})$ \\
Dose 500 (Tempos) & $13,86^{* *}$ & 0,942 & $\mathrm{Y} 2=3,856-0,485(\mathrm{X})$ \\
Dose 1000 (Tempos) & $75,14^{* *}$ & 0,990 & $\mathrm{Y} 3=3,793-1,130(\mathrm{X})$ \\
Dose 1500 (Tempos) & $84,19 * *$ & 0,990 & $\mathrm{Y} 4=3,289-1,196(\mathrm{X})$ \\
\hline
\end{tabular}

(1) Significativo ao nível de $82 \%$

(2) Significativo ao nível de $93 \%$

(3) Significativo ao nível de $90 \%$ 
Tabela 3 - Efeitos de doses de IBA dentro de tempos para as variáveis estudadas.

\begin{tabular}{|c|c|c|c|}
\hline Desdobramento & $\mathbf{F}$ & $\mathrm{r}^{2}$ & Equação Ajustada \\
\hline \multicolumn{4}{|l|}{ Var. Peso Seco } \\
\hline T. Rápido (Doses) & $5,09 * *$ & 0,743 & $Y 1=1,647+0,000171 X$ \\
\hline T. 1 hora (Doses) & $15,98 * *$ & 0,779 & $\mathrm{Y} 2=1,777+0,000934(\mathrm{X})-6,8 \mathrm{E}-7(\mathrm{X} 2)$ \\
\hline T. 2 horas (Doses) & $8,53 *$ & 0,818 & $Y 3=1,701+0,000476(X)-4,9 E-7(X 2)$ \\
\hline \multicolumn{4}{|c|}{ Var. Comp. Rafzes } \\
\hline T. Rápido (Doses) & $2,67^{(1)}$ & 0,998 & $Y 1=22,463+0,0008675(X)-6,7 E-6(X 3)$ \\
\hline T. 1 hora (Doses) & $27,61 * *$ & 0,955 & $\mathrm{Y} 2=27,150-0,0097(\mathrm{X})$ \\
\hline T. 2 horas (Doses) & $78,92^{* *}$ & 0,953 & $\mathrm{Y} 3=28,175-0,0164(\mathrm{X})$ \\
\hline \multicolumn{4}{|l|}{ Var. Notas } \\
\hline T. Rápido (Doses) & $5,89 *$ & 1,000 & $Y 1=3,565+0,001157(X)-8,6 E-(X 2)$ \\
\hline T. 1 hora (Doses) & $82,43 * *$ & 0,975 & $\mathrm{Y} 2=4,205-0,001583(\mathrm{X})$ \\
\hline T. 2 horas (Doses) & $161,66^{*}$ & 0,979 & $\mathrm{Y} 3=4,092-0,002216(\mathrm{X})$ \\
\hline
\end{tabular}

(1) Significativo ao nivel de $82 \%$

(2) Significativo ao nivel de $93 \%$

(3) Significativo ao nivel de $90 \%$

Os tempos de imersão com $500 \mathrm{ppm}$ de IBA proporcionaram os maiores ganhos de matéria seca, com máximo verificado para imersão durante $1,1 \mathrm{~h}, \mathrm{o}$ que difere do efeito desse tratamento sobre as outras variaveis, onde o aumento do tempo de imersão foi prejudicial. $\mathrm{Na}$ ausencia de IBA o ganho de peso seco foi genericamente inferior ao observado nas dosagens de 500 e $1.000 \mathrm{ppm}$, além do que não se observou efeito significativo do tempo de imersão sobre a produção de materia seca.

$\mathrm{Na}$ ausência de IBA, a imersão em água não proporcionou maior peso seco, observando-se porém um aumento no comprimento das raizes e melhor aspecto visual, o que poderia ser atribuido a maior produção e/ou translocação de auxinas endógenas, associados à redistribuição de elaborados e não necessariamente ao acúmulo de biomassa, traduzindo-se por um melhor enraizamento sem o correspondente aumento de materia seca.

As variáveis notas e comprimentos da maior raiz (Tabela 3 e Figura 2) mostram idêntico comportamento para todas as combinações de tratamento rápido e se mostraram mais eficiente para dosagens de IBA entre 600 e 700 ppm, com pequena variação entre 0 e $1.500 \mathrm{ppm}$. $O$ efeito de doses crescentes de IBA tanto em tratamentos por $1 \mathrm{~h}$, quanto por $2 \mathrm{~h}$, foi linearmente decrescente, sendo mais acentuado para $2 \mathrm{~h}$ de imersão, o que caracteriza perfeitamente a ação prejudicial do incremento das dosagens aliado ao incremento do tempo de imersão.

Para a variavel peso seco o aumento das dosagens dentro do tratamento rápido levou a aumentos linearmente crescentes, indicando que melhores resultados poderiam ser obtidos com dosagens superiores a $1.500 \mathrm{ppm}$ de IBA. Na avaliação das doses de IBA para $1 \mathrm{~h}$ de imersão produziu-se genericamente o maior peso seco comparadamente aos outros tempos de imersão, com ponto de maxima em aproximadamente $700 \mathrm{ppm}$. No tratamento por $2 \mathrm{~h}, 0$ máximo foi alcançado com aproximadamente $500 \mathrm{ppm}$, observando-se um efeito negativo a partir daí.

Maiores tempos de imersão em IBA proporcionaram resultados em geral prejudiciais para o enraizamento, o que reforça a hipótese da existência de auxina endogena em quantidade suficiente para o enraizamento e o subsequente retomada do crescimento, observados com maior clareza nas variáveis notas e comprimento da maior raiz, embora o efeito sobre o peso seco tenha sido favorecido pelo tratamento por $1 \mathrm{~h}$.

Considerando-seo processo de propagação de plantas por estaquia, observa-se nas primeiras faces do estaqueamento, que o desenvolvimento ocorre às 

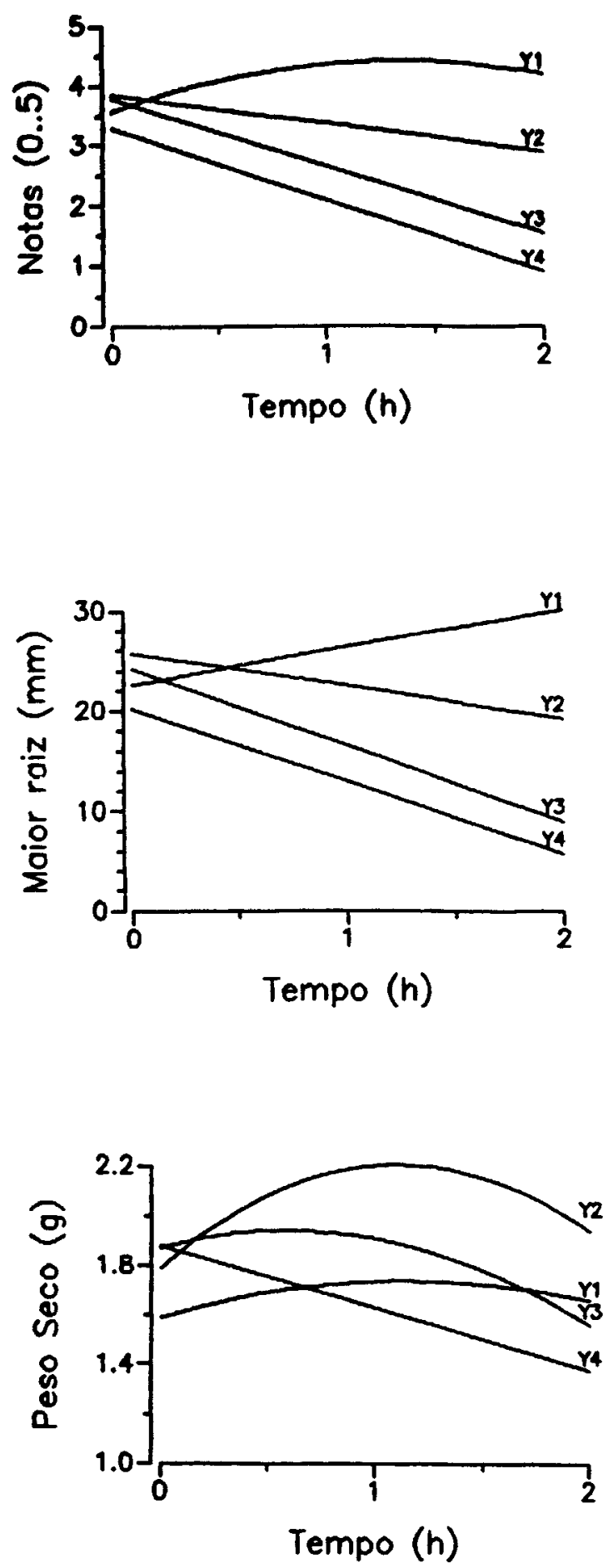

Figura 1 - Efeitos do tempos de imersão dentro das doses de IBA para as variáveis notas, comprimento da maior raíz e peso seco $(\mathrm{Y} 1=0 \mathrm{ppm} ; \mathrm{Y} 2=500 \mathrm{ppm} ; \mathrm{Y} 3=1.000 \mathrm{ppm} ; \mathrm{Y} 4=1.500 \mathrm{ppm})$. 

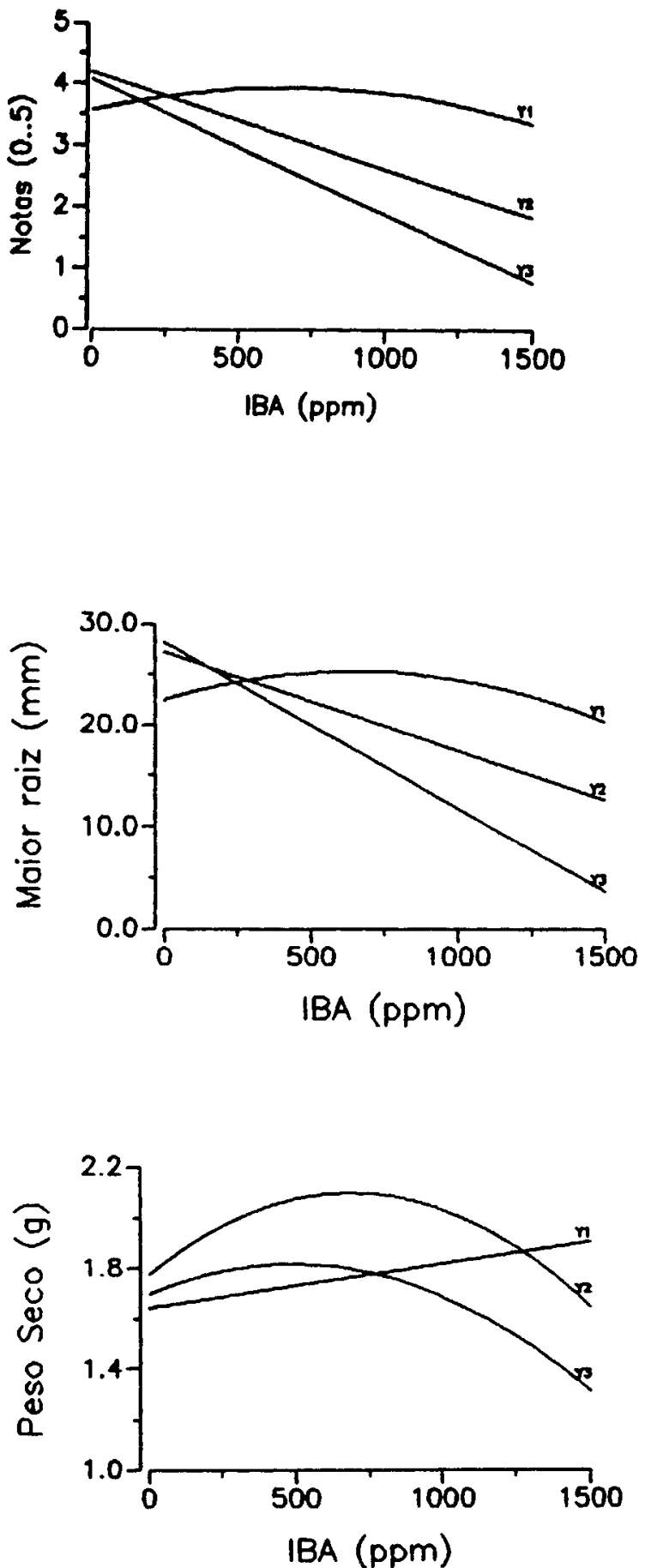

Figura 2 - Efeitos das doses de IBA dentro dos tempos de imersão para as variáveis notas, comprimento da maior raiz e peso seco $(\mathrm{Y} 1=5 \mathrm{~s} ; \mathrm{Y} 2=1 \mathrm{~h} ; \mathrm{Y} 3=2 \mathrm{~h})$. 
expensas das reservas, reduzindo a materia seca total, até que o sistema radicular em estabelecimento permita a redução gradual destas perdas levando ao acúmulo de matéria seca. Em contrapartida, o comprimento das raízes manifesta-se de forma crescente durante praticamente todo o desenvolvimento vegetal; dessa maneira as variáveis apresentam comportamento diferenciado, como os obtidos no experimento. Assim a escolha da variável que melhor represente o processo de enraizamento depende ainda da análise do crescimento da planta.

\section{CONCLUSÕES}

1. Existe interação altamente significativa entre as dosagens de IBA e os tempos de imersão.

2. A variável notas retrata de forma bastante próxima o comprimento da maior raíz, tornando dispensável o estudo desta última variável, em virtude da pouca praticidade e maiores erros associados.

3. O melhor aspecto visual foi verificado na ausência de IBA, em imersão por 1,3 h. Na presença de IBA o aumento do tempo de imersão foi prejudicial.

4. Caso se opte por um tratamento rápido, o melhor aspecto visual é obtido por dosagens entre 600 e 700 ppm.

5. Para se obter mudas de bom aspecto visual, deve-se evitar a combinação de tratamentos prolongados com as maiores dosagens de IBA.

6. A produção de peso seco é maior nas dosagens entre 500 e 700 ppm de IBA por cerca de 1 h de imersão. A ausência de IBA mostrou-se mais eficiente para tratamentos prolongados.

7. Caso se opte por um tratamento rápido, recomendase utilização de dosagens superiores a $1.500 \mathrm{ppm}$, para obtenção de maior peso seco.

8. Sugere-se o estudo de interações entre tempos de imersão entre 5 s e $1 \mathrm{~h}$ com dosagens entre 0 e 700 ppm de IBA.

9. Sugere-se o estudo do desenvolvimento de plantas enraizadas afim de determinar qual ou quais dentre as variáveis melhor representa a qualidade do enraizamento de estacas de crisântemo.

\section{REFERÊNCIAS BIBLIOGRÁFICAS}

ADRIANCE, G.W. \& BRISON, F.R. Cuttage. In: ADRIANCE, G.W. \& BRISON, F.R. Propagation of horticultural plants. New York, McGraw Hill, 1939. p.128-129.
CEAGESP - Companhia de Entrepostos e Armazéns Gerais de São Paulo. Boletim Anual, 1989.

CUTTER, E.G. Anatomia e morfologia vegetal. 2.ed. São Paulo, Roca, 1986. Parte I. 304p.

HARTMANN, H. \& KESTER, D.E. Propagación de plantas, princípios y practicas. México, Continental, 1976. 810p.

JANICK, J. A Ciência da Horticultura. 2.ed. Rio de Janeiro, Freitas Bastos, 1968. 485p.

LEMAIRE, P. Mis crisantemos. Barcelona, Gustavo Gil, 1964. 36p.

SEMANANDA, N.; ORMROD, D.P. \& ADEDIPE, N.O. Rooting of chrysanthemum stem cuttings as affected by 2-chlorethyl phosphonic acid and indolebutyric acid. Ann. Bot. 36:961-5, 1972.

TAKEYOSHI, N.I.; ANRAKI, R.N.; MINAMI, K. \& LIMA, A.M.L.P. Efeitos de diversos substratos no enraizamento de estacas de Chrysanthemum morifolium cv. Polares. In: CONGRESSO BRASILEIRO DE FLORICULTURA E PLANTAS ORNAMENTAIS; 1 ., Rio de Janeiro, 1983. p.137-142. Anais.

WODECKI, M.J. \& HOLCOMB, J. Varying concentration of IBA affects rooting of chrysanthemum cuttings $\mathrm{cv}$. Bright Golden. Hort.Abst. 59:11, 1989. Abstract.

Entregue para publicação em 20.08.91

Aprovado para publicação em 31.10.91 